\title{
Extending Korf's ideas on the pursuit problem
}

\author{
Juan Reverte, Francisco Gallego, Faraón Llorens \\ \{jreverte, fgallego, faraon\}@dccia.ua.es \\ Department of Computer Science and Artificial Intelligence \\ University of Alicante
}

Summary. The prey-predator pursuit problem is referenced many times in literature. It is a generic multi-agent problem whose solutions could by applied to many particular instances. Solutions proposed usually apply non-supervised learning algorithms to train prey and predators. Most of these solutions criticize the greedy algorithm originally proposed by Korf. However, we believe that the improvement obtained by these new proposals does not pay off with relation to their complexity.

The method used by Korf is a natural way to surround a prey without explicit communication between predators. The knowledge one predator has about others is limited just to what it can see. In Korf's model, agents are able to see the complete world at once. In this paper we propose to start from Korf's ideas and extend them to improve his model. First, we propose a simple extension of Korf's fitness function and we consider the problems related to a partial view of the world. Second, we propose a communication protocol to partially overcome them. The final results suggest that more work needs to be done, and we propose a way to follow-on.

Keywords: Prey-predator, multi-agent systems, communication

\subsection{Introduction}

The Prey-predator pursuit problem emerges every time that a group of agents have to chase and surround another agent (or other agents) that tries to evade them [1]. The goal of the predator agents is to surround prey(s) without touching it, arriving to a final state position where predators impede any possible orthogonal movement to the prey, whilst the goal of the prey, as expected, is not to be captured.

This problem has been addressed many times in the literature from diferent points of view [2][3][4][5][7].The first accepted solution was the one proposed by Korf [7]. Korf just combined 2 forces in the way that each predator is "attracted" by the prey and "repelled" from the closest predator. This solution keeps predators away from other predators while they get closer to the prey, thus chasing the prey like a circle stretching, in the best case. Korf's simple solution has got a lot of criticism and a great number of other solutions and 
alternatives have emerged. For instance, Haynes [3] used genetic programming to evolve coordinated behaviours of predators. Haynes compared diferences between communicating and non-communicating predators with respect to their success chasing the prey. He also co-evolved predators and the prey and found that a prey always going straight in diagonal is never caught by predators unless it is slower than them. Chainbi [2] used petri nets to coordinate predators while solving concurrency problems between them, while Jim and Giles [4] used a genetic algorithm and multi-agent communication using a blackboard. One of the most interesting alternatives was the one proposed by Katayama et al. [5]. They used an agent-oriented reinforcement learning algorithm, namely profit-sharing, with analytic hierarchy process (AHP) integrated. Their idea was to introduce primary knowledge to guide the agents when they start the learning process. It is a great idea to give some knowledge as a kind of "hints" to the agents when they start learning, but it seems not to be reasonable to continue giving these "hints" once agents have grown and developed their own knowledge. The solution proposed by Katayama et al. is to progresively take back the "hints", leaving the agents finally with their own knowledge. Analyzing the results shown by these alternatives to Korf [7] we conclude that they are not enough to state that Korf's ideas were not right. Korf idea was really simple: determine the next movement of each predator with a fitness function dependant on distance to the prey and distance to the nearest other predator. The fitness function simulated an "attractive force" to the prey and a "repulsive force" from the nearest predator. This idea represents the starting point of this paper. Section 2 explains the extensions we propose to Korf's ideas. Section 3 shows our implementation and results. Finally, section 4 sums up our conclusions and next under-development proposals.

\subsection{Extending Korf's ideas}

Originally, Korf used a 100x100 discrete grid where all agents must occupy distinct positions. He stablished a rotatory turn system for the agents. At every turn one agent can move to an empty neighboring cell or remaining stationary. $90 \%$ of the times, prey moved to the neighboring cell that is furthest away from the nearest predator, remaining stationary the other $10 \%$. The prey begins in the center of the board, and the initial position of the predators is randomly generated.

The first and simplest extension we propose to Korf's model is to change the fitness function to make each predator repel from all other predators it has in its viewing field. This should improve behaviour of predators when they are close, for instance, when they are on the point of catching the prey. Equation 1.1 shows the extended fitness function. This fitness function is calculated for each possible cell where the predator is able to move to, finally selecting the cell with maximum fitness. We assume $X_{p}, Y_{p}$ as the location of the prey, 
$X_{i}, Y_{i}$ as the location of predator $i$, and $d\left(x_{1}, y_{1}, x_{2}, y_{2}\right)$ as the manhattan distance between two cells.

$$
f(x, y)=d\left(x, y, X_{p}, Y_{p}\right)-k \sum_{i=1}^{n} d\left(x, y, X_{i}, Y_{i}\right)
$$

In Korf's model, the most influent initial condition was the ability of agents to see the complete board. This is what makes his solution possible, but it is almost never happening in a real or virtual environment. It is common for agents to have a limited view of the environment as it would be the case in a real environment. In this more real situation, Korf's ideas are still interesting, but need to be extended and supported with communication between agents [4]. In our model, we consider that an agent located at $x, y$ with a field of vision (FOV) of $n$ cells means the agent is only able to preceive what happens in cells $\left\{\left(x^{\prime}, y^{\prime}\right) / x-n<=x^{\prime}<=x+n, y-n<=y^{\prime}<=y+n\right\}$. Take into account that an agent is only able to communicate with thouse inside its FOV.

This new situation does not prevent Korf's algorithm from catching the prey. However, the algorithm becomes slower. Namely, it takes more time to predators to find the prey. In strict sense, the probability of a predator indefinitely not finding the prey is not 0 , and that is definitely a problem to overcome. Our propose is a simple yet effective protocol for communicating prey sights we have called Cascading Sight Notice (CSN, see figure 1.1). This protocol starts when a predator sees the prey. The predator sends a message to others in its FOV, telling them the relative location of the prey from its point of view. The hearing predators make an addition of the two vectors and then get aware of the location of the prey. These predators, once they have located the prey with respect to their own place, resend the message to other predators. The cycle continues until no predator is hearing or hearing predators already know where prey is.

\subsection{Results}

To validate our approach we compared it with Korf's method in different simulated environment conditions. For running the simulations we used Kok\&Vlassis' Pursuit Domain [6]. This environment simulates a discrete, toroidal predator-prey environment with several configurable characteristics. Concretely, we used a 30x30 cells field, allowing agents to move diagonal, with the prey starting on the center and predators starting randomly placed. We lauched 4 predators to capture 1 prey. Finally, in case of collision, only predators colliding were penalized. Simulation was always ran for 250 consecutive episodes, and we got the average results.

With this configuration we ran 2 groups of 4 experiments each. Each group refers to a differently behaving prey whilst each experiment is about differently behaving predators. The 2 preys were: g1) randomly moving prey, g2) prey 

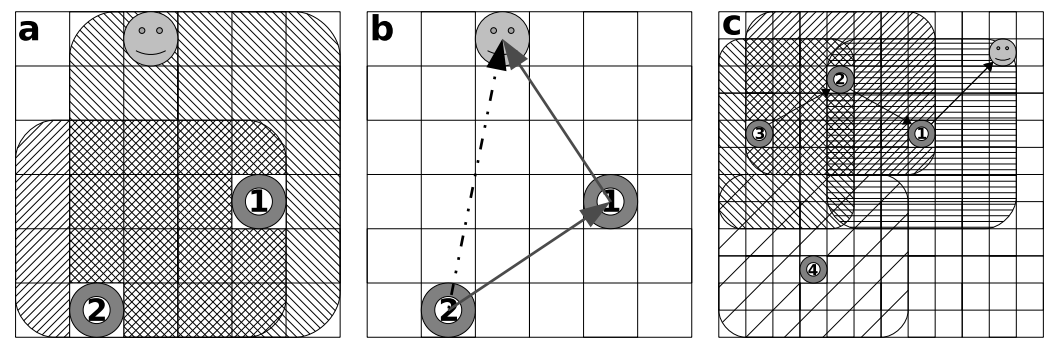

Fig. 1.1. a) Predator 1 sees prey, pred.2 sees pred.1. b) Pred.2 calculates prey location using pred.1 info. c) Preds.1, 2, 3 can figure out prey location, pred.4 cannot

that moves to the cell most distant from menacing predators. The experiments ran were: e1) Korf's predators, e2) extended Korf's predators (using equ. 1.1), e3) Korf's predators using CSN, e4) extended Korf's predators using CSN.

Figure 1.2 shows results from the 8 experiments. We ran each varying the FOV from a minimum of 3 cells to a complete FOV of 15 cells (15 to each direction of a toroidal world covers the 30x30 field). Results show that our extended version of Korf's algorithm represents an improvement of an order of magnitude in most cases, with independence of the type of prey. This improvement is comparable to the ones achieved by most of the cited works in this paper. It is really curious to compare Korf's and Extended
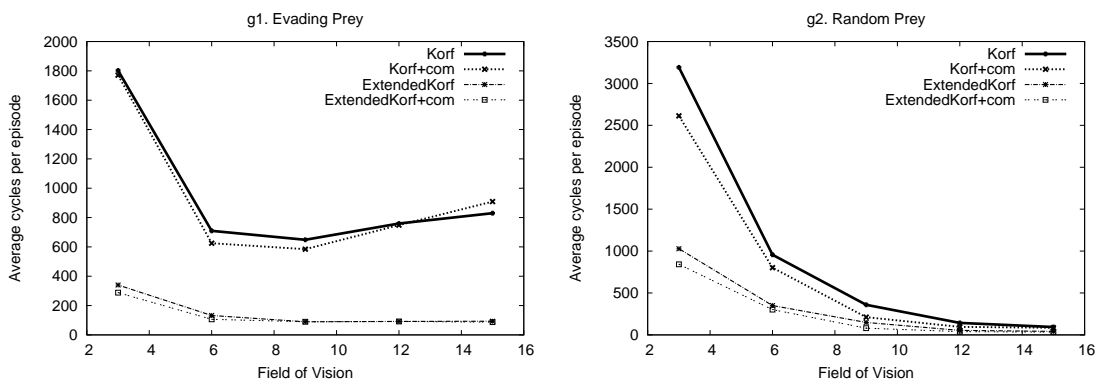

Fig. 1.2. Comparison between Korf's and Ext. Korf's models, with and without interagent communication

Korf's algorithms with their versions including CSN protocol described in section 1.2. Results show that CSN represents a small improvement that is only significant depending on type of prey and FOV. When FOV is complete, CSN is unnecesary; when FOV is at the bare minimum, CSN significance depends on the prey. This is due to the chasing movements predators do with the evading prey and the necessity of being inside FOV to communicate with others. The bigger the world, the more disperse predators are and the more difficult to communicate. 
Notice that PDP treats collisions randomly replacing predators which collide and uses a toroidal world. These are two main differences between this work and Korf's that should be considered to compare results.

\subsection{Conclusions and Futher Work}

The Predator-prey pursuit problem is considered a good testbed for multiagent collaboration. A great number of solutions have been presented. Recent solutions focus on machine learning and criticize Korf's greedy approach.

Although recent works have shown great results, we believed that Korf's idea still has a good potential to explore. Therefore, we proposed and developed a simple extension of Korf's algorithm. Moreover, as Korf did not take into account limited FOV of the agents, we also have developed a simple protocol (CSN) to overcome part of the problems caused by this limited FOV.

Our results show that with these two simple extensions, Korf's ideas get results comparable to most machine learning aproaches cited here. This supports our hipothesis that Korf's idea has a great potential. However, CSN is pretty simple and does not seem to be enogh. As future work we plan to develop a protocol to improve predators early finding and noticing the prey. This new protocol will also deal with concurrency problems and prevent collisions.

\section{References}

1. M. Benda, V. Jagannathan, and R. Dodhiawalla. On optimal cooperation of knowledge sources. Technical Report Tech. Rep. BCS-G2010-28, Boeing AI Center, Boeing Computer Services, Bellevue, WA, 1986.

2. W. Chainbi, C. Hanachi, and C. Sibertin-Blanc. The Multi-agent Prey-Predator problem : A Petri net solution. In Proceedings of the IMACS-IEEE-SMC conference on Computational Engineering in Systems Application (CESA'96), pages 692-697, Lille, France, 1996.

3. Thomas Haynes and Sandip Sen. Evolving behavioral strategies in predators and prey. In Sandip Sen, editor, IJCAI-95 Workshop on Adaptation and Learning in Multiagent Systems, pages 32-37, Montreal, Quebec, Canada, 20-25 1995. Morgan Kaufmann.

4. Kam-Chueun Jim and C. Lee Giles. Talking helps: Evolving communicating agents for the predator-prey pursuit problem. Artificial Life, 6(3):237-254, 2000.

5. Kengo Katayama, Takahiro Koshiishi, and Hiroyuki Narihisa. Reinforcement learning agents with primary knowledge designed by analytic hierarchy process. In SAC '05: Proceedings of the 2005 ACM symposium on Applied computing, pages 14-21, New York, NY, USA, 2005. ACM.

6. Jelle R. Kok and Nikos Vlassis. The pursuit domain package. Technical Report Technical Report IAS-UVA-03-03, Informatics Institute, University of Amsterdam, The Netherlands, August 2003.

7. Richard E. Korf. A simple solution to pursuit games. In Proceedings of the 11th International Workshop on Distributed Artificial Intelligence, pages 183194, Glen Arbor, MI., February 1992. 